\title{
Exact quantum master equation for a molecular aggregate coupled to a harmonic bath
}

\author{
Maxim F. Gelin, ${ }^{1}$ Dassia Egorova, ${ }^{2}$ and Wolfgang Domcke ${ }^{1}$ \\ ${ }^{1}$ Department of Chemistry, Technische Universität München, D-85747 Garching, Germany \\ ${ }^{2}$ Institute of Physical Chemistry, Christian-Albrechts- \\ Universität zu Kiel, D-24098 Kiel, Germany
}

\begin{abstract}
We consider a molecular aggregate consisting of $N$ identical monomers. Each monomer comprises two electronic levels and a single harmonic mode. The monomers interact with each other via dipole-dipole forces. The monomer vibrational modes are bilinearly coupled to a bath of harmonic oscillators. This is a prototypical model for the description of coherent exciton transport, from quantum dots to photosynthetic antennae. We derive an exact quantum master equation for such systems. Computationally, the master equation may be useful for the testing of various approximations employed in theories of quantum transport. Physically, it offers a plausible explanation of the origins of long-lived coherent optical responses of molecular aggregates in dissipative environments.
\end{abstract}




\section{INTRODUCTION}

Quantum systems coupled to a "bath" of harmonic oscillators are paradigmatic models in nonequilibrium quantum statistical mechanics [1 6]. The primary goal of the theory of quantum dissipative systems is the derivation of so-called master equations, which govern the dynamics of the reduced density matrix of a "system" of interest, starting from the Liouville - von Neumann equation for the combined system+bath ensemble. A well know example is Redfield theory (or modifications thereof [7]), which is valid in the limit of weak system-bath

coupling [1-8]. A master equation can also be derived in the limit of the strong system-bath coupling [9, 10]. More advanced treatments have lead to hierarchies of master equations [11], which are valid beyond weak system-bath coupling and the Markovian approximation.

With these methods, the time evolution of the reduced density matrix can be computed to high accuracy by the solution of truncated infinite chains of equations for auxiliary density matrices.

There exist very few system-bath models for which "exact" closed-form quantum master equations have been derived. A master equation is called exact if the time evolution of all operators representing observables of the system is identical with the time evolution described by the Liouville - von Neumann equation for the system+bath ensemble. An obvious example is the so-called phase-noise case, in which the system Hamiltonian commutes with the system-bath coupling [12]. Nontrivial exact quantum master equations are known for the free particle, the harmonic oscillator, as well as for two or $N$ identical oscillators bilinearly coupled to the harmonic bath [13-19]. The system-bath Hamiltonians that are quadratic in Bose or Fermi operators also allow for an analytical description of the system dynamics (see, e.g., [20 22] ]).

In the present communication, we present the derivation of an exact quantum master equation for a molecular aggregate consisting of $N$ identical molecular monomers. Each monomer comprises two electronic levels and a single harmonic vibrational mode, which is coupled to a harmonic bath. This model is prototypical for the description of the transport of excitons, from quantum dots to photosynthetic antennae and DNA [4, 23-27]. Apart from curiosity (as R. J. Baxter put it [28], “... the model is relevant and it can be solved, so why not to do so and see what it tells us?") our motivation is threefold. Conceptually, the derived master equation is, to our knowledge, the first exact master equation for a system with 
electronic inter-monomer couplings, intra-monomer electron-vibrational couplings as well as vibrational dissipation. None of these couplings are assumed to be weak. Computationally, the exact master equation may be useful for the testing of various approximations (e.g., weak system-bath coupling, or weak electron-vibrational coupling, or weak inter-monomer coupling) which are frequently employed in theories of quantum transport [4, 24-27, 29-31]. Fundamentally, the master equation offers a plausible explanation of the origins of long-lived coherent optical responses of molecular aggregates in dissipative environments [32-41].

We use units in which $\hbar=1$.

\section{DERIVATION OF THE MASTER EQUATION}

Consider the total Hamiltonian $H$, which consists of the system (S) Hamiltonian, the bath (B) Hamiltonian, and their coupling,

$$
H=H_{S}+H_{B}+H_{S B}
$$

The system comprises $N$ electronic two-level systems each of which possesses a vibrational mode:

$$
H_{S}=H_{e x}\left(B_{a}^{\dagger}, B_{a^{\prime}},\left|X_{j}-X_{j^{\prime}}\right|\right)+\sum_{k=1}^{N}\left(\frac{P_{k}^{2}}{2 M}+\frac{M \Omega^{2} X_{k}^{2}}{2}+\xi B_{k}^{\dagger} B_{k} X_{k}\right) .
$$

Here, $B_{a}^{\dagger}$ and $B_{a^{\prime}}$ are the exciton creation and annihilation operators obeying the Pauli commutation rules $\left[B_{a}, B_{a^{\prime}}^{\dagger}\right]=\delta_{a a^{\prime}}\left(1-2 B_{a}^{\dagger} B_{a^{\prime}}\right) . X_{k}, P_{k}=-i d / d X_{k}, M$, and $\Omega$ denote the positions, momenta, masses, and frequencies of the harmonic oscillators. The parameter $\xi$ controls the strength of the intra-monomer exciton-vibrational coupling. The excitonic Hamiltonian $H_{e x}$ may depend parametrically on the distances $\left|X_{j}-X_{j^{\prime}}\right|$. We need not specify $H_{e x}$, but we require that it conserves the number of excitons and thus commutes with the number operator $\hat{N}$ :

$$
\left[H_{e x}, \hat{N}\right]=0, \hat{N} \equiv \sum_{k=1}^{N} B_{k}^{\dagger} B_{k}
$$

We allow for variable couplings between the identical monomers, since the monomers are fixed (e.g., in protein matrices) and interact via (usually, dipole-dipole) forces, which depend on the relative positions and orientations of the monomers. 
The system interacts with a harmonic bath via bilinear system-bath coupling

$$
H_{S B}+H_{B}=\sum_{i=1}^{N_{B}}\left(\frac{p_{i}^{2}}{2 m_{i}}+\frac{m_{i} \omega_{i}^{2}}{2} \sum_{k=1}^{N}\left(x_{i}-\frac{c_{i} X_{k}}{m_{i} \omega_{i}^{2}}\right)^{2}\right) .
$$

Here $x_{i}, p_{i}=-i d / d x_{i}, m_{i}$, and $\omega_{i}$ denote the positions, momenta, masses, and frequencies of the bath oscillators, and $c_{i}$ are the system-bath coupling coefficients. All $N$ monomers are assumed to be identical, and the influence of the bath (4) on the system dynamics is determined by a single spectral density

$$
g(\omega)=\frac{\pi}{2} \sum_{i=1}^{N_{B}} \frac{c_{i}^{2}}{m_{i} \omega_{i}} \delta\left(\omega_{i}-\omega\right)
$$

Otherwise, the masses $M$, frequencies $\Omega$, electronic couplings $\xi$, and system bath-coupling coefficients $c_{i}$ acquire a $k$-dependence, and an exact master equation cannot be derived. However, the master equation derived below remains true for different oscillators provided $M_{k} \sim \Lambda_{k} \sim \xi_{k} \sim c_{i, k}$ for any $k$.

In the description of energy-transport and relaxation phenomena in excitonic systems, the vibrational bath is usually assumed to couple electronic degrees of freedom directly, inducing fluctuations of the site energies [26, 27]. In the present study, as well as, e.g., in Refs. [4, 42, 43], each monomer is coupled to the bath through its vibrational reaction mode. As has been shown in [44-48], the two methods are, in principle, equivalent: By introducing an appropriate canonical transformation, one can switch from one description to another by incorporating the system modes into the bath or by singling-out several (high-frequency) modes from the bath and treating them explicitly. The present choice of the system-bath coupling through the monomer vibrational modes is motivated by three major reasons. First, it allows us to derive an exact master equation. Second, the explicit treatment of high frequency modes strongly coupled to electronic two-level systems allows us to assume that the remaining bath modes are coupled to the monomers rather weakly. This may become important for the extension of the present theory beyond the system-bath Hamiltonian (44). Third, the explicit consideration of high-frequency vibrational modes facilitates the study of vibrationally-coherent effects in the system responses and spectroscopic signals (see, e.g., [4, 42, 43]).

Let us introduce new variables specifying the system oscillators, the center-of-mass coor- 
dinate $R$ and the internal coordinates $Q_{j}$

$$
R=\frac{1}{N} \sum_{j=1}^{N} X_{j} ; \quad Q_{j}=X_{j+1}-X_{j}, \quad j=1,2, \ldots, N-1 .
$$

The transformation ([6]) is described by the $N \times N$ matrix $S$ :

$$
Q_{j}=\sum_{k=1}^{N} S_{j k} X_{k}, \quad R=\sum_{k=1}^{N} S_{N k} X_{k}
$$

The transformation back to the original coordinates $X_{k}$ is given by the inverse matrix:

$$
X_{j}=\sum_{k=1}^{N-1}\left(S^{-1}\right)_{j k} Q_{k}+\left(S^{-1}\right)_{j N} R
$$

We were unable to derive an explicit analytical expression for $S^{-1}$ for arbitrary $N$ (for any finite $N$ this can be done numerically). For the following derivations, it is sufficient to realize that $S^{-1}$ obeys the conditions

$$
\sum_{j=1}^{N}\left(S^{-1}\right)_{j k} \equiv 0, \quad(k=1,2, \ldots, N-1) ; \quad\left(S^{-1}\right)_{j N} \equiv 1, \quad(j=1,2, \ldots, N),
$$

which are elementary consequences of Eq. (8). According to Eq. (66), the original momenta are connected to the new momenta $P_{R} \equiv-i d / d R$ and $P_{Q, j} \equiv-i d / d Q_{j}$ as follows:

$$
P_{j}=\frac{1}{N} P_{R}-P_{Q, j}+P_{Q, j-1}
$$

The change of the system vibrational variables (6) is borrowed from the theory of Gaussian polymers (see, e.g., [49]). It is linear, non-singular ( $\operatorname{det} S=1$ ), but not canonical. The transformation (6) is just a technical tool, and we can return to the original canonical $X_{j}$ representation at the end of the derivation. The choice (6) of the internal coordinates is natural for a linear array of monomers. Other choices may be preferable in different situations. The transformation ([6]) can also be applied to the derivation of the master equation for $N$ identical oscillators bilinearly coupled to a harmonic bath, generalizing thereby the approach of Ref. [19].

By insertion of Eqs. (8) and (10) into Eqs. (2) and (4), we obtain the system Hamiltonians in the new variables

$$
\begin{gathered}
H_{S}=H_{S}^{(R)}+H_{S}^{(Q)} \\
H_{S}^{(R)}=\left(\frac{P_{R}^{2}}{2 M N}+\frac{M N \Omega^{2} R^{2}}{2}+\xi N \hat{N} R\right),
\end{gathered}
$$


$H_{S}^{(Q)}=H_{e x}\left(B_{a}^{\dagger}, B_{a^{\prime}}, Q_{j}\right)+\sum_{k=1}^{N} \frac{\left(P_{Q, k-1}-P_{Q, k}\right)^{2}}{2 M}+\sum_{k, l=1}^{N-1} G_{k l} \frac{M \Omega^{2} Q_{k} Q_{l}}{2}+\sum_{k=1}^{N} \sum_{l=1}^{N-1} \xi B_{k}^{\dagger} B_{k}\left(S^{-1}\right)_{k l} Q_{l}$

(by definition, $P_{Q, 0}=P_{Q, N} \equiv 0$ ). Here $\hat{N}$ is the exciton number operator (3) and

$$
G_{k l}=\sum_{j=1}^{N}\left(S^{-1}\right)_{j k}\left(S^{-1}\right)_{j l}
$$

The bath and the system-bath coupling Hamiltonians also split into contributions associated with the variables $R$ and $Q$

$$
H_{S B}+H_{B}=H_{S B}^{(R)}+H_{S B}^{(Q)}
$$

where

$$
H_{S B}^{(R)}=\sum_{i=1}^{N_{B}}\left(\frac{p_{i}^{2}}{2 m_{i}}+N \frac{m_{i} \omega_{i}^{2}}{2}\left(x_{i}-\frac{c_{i} R}{m_{i} \omega_{i}^{2}}\right)^{2}\right)
$$

and

$$
H_{S B}^{(Q)}=\sum_{k, l=1}^{N-1} G_{k l} \frac{\Lambda_{B} Q_{k} Q_{l}}{2}
$$

Here

$$
\Lambda_{B}=\sum_{i=1}^{N_{B}} \frac{c_{i}^{2}}{m_{i} \omega_{i}^{2}},
$$

so that $H_{S B}^{(Q)}$ is the bath-induced renormalization of the potential energy (the so-called Lamb-shift). Combining the Hamiltonians, we have

$$
\begin{gathered}
H=H_{\Sigma}^{(R)}+H_{\Sigma}^{(Q)} \\
H_{\Sigma}^{(R)} \equiv H_{S}^{(R)}+H_{S B}^{(R)}, \quad H_{\Sigma}^{(Q)} \equiv H_{S}^{(Q)}+H_{S B}^{(Q)}
\end{gathered}
$$

(the subscript $\Sigma$ indicates total system+bath Hamiltonians). Due to the requirement (3) the Hamiltonians (19) commute

$$
\left[H_{\Sigma}^{(R)}, H_{\Sigma}^{(Q)}\right]=0
$$

Equations (19)-(21) summarize the first main result of the present paper. In words, they show that the parent Hamiltonian (1) can be transformed into the Hamiltonian (19), which is the sum of two mutually commuting Hamiltonians, $H_{\Sigma}^{(R)}$ and $H_{\Sigma}^{(Q)}$. $H_{\Sigma}^{(Q)}$ depends on the internal coordinates $Q_{j}$ and is independent of the bath variables. The bath enters $H_{\Sigma}^{(Q)}$ only through the bath-induced potential, $H_{S B}^{(Q)} . H_{\Sigma}^{(R)}$ depends on the center-of-mass coordinate $R$, through which the system is bilinearly coupled to the bath. The discrete degrees of 
freedom enter $H_{\Sigma}^{(R)}$ exclusively via the number operator $\hat{N}$. After the expansion in the eigenfunctions of $\hat{N}$, the Hamiltonian $H_{\Sigma}^{(R)}$ becomes harmonic. It is this latter fact which allows us to exactly integrate the bath out and derive an exact master equation.

For doing that, let us introduce the total (system+bath) density matrix $\tilde{\rho}(t)$, which obeys the Liouville-von Neumann equation

$$
\partial_{t} \tilde{\rho}(t)=-i\left[H_{\Sigma}^{(R)}+H_{\Sigma}^{(Q)}, \tilde{\rho}(t)\right]
$$

Since the Hamiltonians $H_{\Sigma}^{(R)}$ and $H_{\Sigma}^{(Q)}$ commute, we can, following Ref. [18], introduce a new density matrix

$$
\partial_{t} \tilde{\rho}^{(R)}(t)=-i\left[H_{\Sigma}^{(R)}, \tilde{\rho}^{(R)}(t)\right]
$$

which is connected to the original density matrix via the unitary transformation

$$
\tilde{\rho}(t) \equiv \exp \left\{-i H_{\Sigma}^{(Q)} t\right\} \tilde{\rho}^{(R)}(t) \exp \left\{i H_{\Sigma}^{(Q)} t\right\}
$$

Since $H_{\Sigma}^{(R)}$ (as well as $H_{\Sigma}^{(Q)}$ ) commutes with the number operator $\hat{N}$, it can be expanded in the eigenfunctions of $\hat{N}$ :

$$
H_{\Sigma}^{(R)} \equiv \sum_{n=0}^{N}|n\rangle\langle n| H_{\Sigma, n}^{(R)} .
$$

Here

$$
H_{\Sigma, n}^{(R)} \equiv\left\langle n\left|H_{\Sigma}^{(R)}\right| n\right\rangle, \hat{N}|n\rangle=n|n\rangle
$$

Therefore, Eq. (23) can be rewritten in the form

$$
\begin{gathered}
\partial_{t} \tilde{\rho}_{m n}^{(R)}(t)=-i H_{\Sigma, m}^{(R)} \tilde{\rho}_{m n}^{(R)}(t)+i \tilde{\rho}_{m n}^{(R)}(t) H_{\Sigma, n}^{(R)}, \\
\tilde{\rho}^{(R)}(t) \equiv \sum_{n, m=0}^{N}|n\rangle\langle m| \tilde{\rho}_{m n}^{(R)}(t) .
\end{gathered}
$$

Suppose that the density matrix at $t=0$ commutes with the number operator $\hat{N}$. Hence it can be expanded in the eigenfunctions of $\hat{N}$ as follows:

$$
\tilde{\rho}^{(R)}(0)=\sum_{n=0}^{N}|n\rangle\langle n| \tilde{\rho}_{n n}^{(R)}(0) .
$$

$\tilde{\rho}_{n n}^{(R)}(0)$ are not limited to pure states: they can represent a linear combination of pure states belonging to the same $n$. The initial condition (28) is adequate for many practical purposes. Usually, the system is in its ground electronic state $(n=0)$ or can be promoted to its first 
excited electronic state $(n=1)$ by a laser pulse. Preparation of higher-order states $(n>1)$ is also possible via multiple and/or strong laser pulses. The level of description based on Eq. (28) is appropriate for describing sequential (strong-pulse) spectroscopic signals, given the pulses are temporally well-separated [50]. If Eq. (28) is not fulfilled, we have to consider Eq. (27) for $n \neq m$. In this case, $\tilde{\rho}_{m n}^{(R)}(t)$ is propagated via the Hamiltonian $H_{\Sigma, n}^{(R)}$ in the bra and via the Hamiltonian $H_{\Sigma, m}^{(R)} \neq H_{\Sigma, n}^{(R)}$ in the ket, and an exact master equation is impossible to derive.

Given the initial condition (28), we restrict ourselves to the consideration of Eq. (27) with $n=m$,

$$
\partial_{t} \tilde{\rho}_{n n}^{(R)}(t)=-i\left[H_{\Sigma, n}^{(R)}, \tilde{\rho}_{n n}^{(R)}(t)\right]
$$

The Hamiltonian $H_{\Sigma, n}^{(R)}(26)$ is the sum of the system Hamiltonian $H_{S}^{(R)}$ (12) (in which me must substitute $\hat{N}$ by its eigenavalue $n$ ) and the system-bath Hamiltonian $H_{S B}^{(R)}(\underline{16})$. Let us now define the renormalized system oscillator mass $\bar{M}=N M$, the renormalized bath oscillator frequencies $\bar{\omega}_{i}^{2}=N \omega_{i}^{2}$, and the renormalized system-bath coupling coefficients $\bar{c}_{i}=N c_{i}$, as well as introduce the shifted center-of-mass coordinate $\bar{R}=R+n \xi /\left(M \Omega^{2}\right)$, and the shifted bath coordinates $\bar{x}_{i}=x_{i}+n \xi c_{i}^{2} /\left(M \Omega^{2} m_{i} \omega_{i}^{2}\right)$. After all these transformations, the Hamiltonian $H_{\Sigma, n}^{(R)}$ assumes the form

$$
H_{\Sigma, n}^{(R)}=H_{\Sigma}^{(\bar{R})} \equiv \frac{P_{R}^{2}}{2 \bar{M}}+\frac{\bar{M} \Omega^{2} \bar{R}^{2}}{2}+\sum_{i=1}^{N_{B}}\left(\frac{p_{i}^{2}}{2 m_{i}}+\frac{m_{i} \bar{\omega}_{i}^{2}}{2}\left(\bar{x}_{i}-\frac{\bar{c}_{i} \bar{R}}{m_{i} \bar{\omega}_{i}^{2}}\right)^{2}\right) .
$$

Apparently, $H_{\Sigma}^{(\bar{R})}$ is $n$-independent; it describes a harmonic oscillator of the mass $\bar{M}$ and frequency $\Omega$ bilinearly coupled to the harmonic bath. The Liouville - von Neumann equation (29) thus becomes

$$
\partial_{t} \tilde{\rho}^{(\bar{R})}(t)=-i\left[H_{\Sigma}^{(\bar{R})}, \tilde{\rho}^{(\bar{R})}(t)\right]
$$

$\tilde{\rho}^{(\bar{R})}(t)$ being the total density matrix in the transformed variables. According to Refs. [1316], the bath can be integrated out in Eq. (31) exactly, yielding the master equation for the reduced density matrix $\rho^{(\bar{R})}(t) \equiv \operatorname{Tr}_{B}\left\{\tilde{\rho}^{(\bar{R})}(t)\right\}$ :

$$
\partial_{t} \rho^{(\bar{R})}(t)=-i\left[H_{S}^{(\bar{R})}, \rho^{(\bar{R})}(t)\right]+\bar{\Re}(t) \rho^{(\bar{R})}(t) .
$$

Here $H_{S}^{(\bar{R})} \equiv P_{R}^{2} /(2 \bar{M})+\bar{M} \Omega^{2} \bar{R}^{2} / 2$ and the dissipation operator is defined as follows:

$$
\begin{aligned}
\bar{\Re}(t) \rho^{(\bar{R})}(t) \equiv & -i a(t)\left[\bar{R}^{2}, \rho^{(\bar{R})}(t)\right]-i b(t)\left[\bar{R},\left\{P_{R}, \rho^{(\bar{R})}(t)\right\}\right] \\
& +c(t)\left[\bar{R},\left[P_{R}, \rho^{(\bar{R})}(t)\right]\right]-d(t)\left[\bar{R},\left[\bar{R}, \rho^{(\bar{R})}(t)\right]\right] .
\end{aligned}
$$


In the above equation, $\{.,$.$\} denotes the anti-commutator, and a(t), b(t), c(t), d(t)$ are real functions which are explicitly defined in [13 16]; they are determined through the spectral density of the renormalized bath introduced in Eq. (30):

$$
\bar{g}(\omega)=\frac{\pi}{2} \sum_{i=1}^{N_{B}} \frac{\bar{c}_{i}^{2}}{m_{i} \bar{\omega}_{i}} \delta\left(\bar{\omega}_{i}-\omega\right)=N g\left(\frac{\omega}{\sqrt{N}}\right)
$$

$(g(\omega)$ is given by Eq. (5) ). Returning to the original variables $R$ yields

$$
\partial_{t} \rho_{n n}^{(R)}(t)=-i\left[H_{S, n}^{(R)}, \rho_{n n}^{(R)}(t)\right]+\Re_{n}(t) \rho_{n n}^{(R)}(t) .
$$

Here $\rho_{n n}^{(R)}, H_{S, n}^{(R)}$, and $\Re_{n}(t)$ are obtained from $\rho^{(\bar{R})}, H_{S}^{(\bar{R})}$, and $\bar{\Re}(t)$ by the replacement of $\bar{R}$ with $R+n \xi /\left(M \Omega^{2}\right)$.

Combining Eqs. (23) and (24) and using Eq. (33), we obtain the desired exact master equation for the reduced (system) density matrix $\rho(t) \equiv \operatorname{Tr}_{B}\{\tilde{\rho}(t)\}$ :

$$
\partial_{t} \rho(t)=-i\left[H_{S}^{(R)}+H_{\Sigma}^{(Q)}, \rho(t)\right]+\Re(t) \rho(t),
$$

$\Re(t) \equiv \sum_{n}|n\rangle \Re_{n}(t)\langle n|$. Equivalently, the master equation can be rewritten as

$$
\partial_{t} \rho(t)=-i\left[H_{S}+H_{S B}^{(Q)}, \rho(t)\right]+\Re(t) \rho(t)
$$

$H_{S B}^{(Q)}$ (Eq. (17)) being the bath-induced renormalization of the system potential. Eqs. (36) and (37) can be transformed back to the original $X_{i}$-representation, if desired. Very similar master equations can be derived assuming that the operators $B_{a}^{\dagger}$ and $B_{a^{\prime}}$ obey either the Bose or the Fermi commutation relations.

From the computational point of view, $H_{S B}^{(Q)}$ cannot be factorized into commuting subHamiltonians for the $Q_{k}$. For small $N$ (see [51] for the vibronic trimer) a suitable matrix representation of $H_{S B}^{(Q)}$ can be introduced. Alternatively, one can invoke the generalized Fulton-Gouterman transformation [52, 53], which diagonalizes $H_{S B}^{(Q)}$ in the electronic Hilbert space, reducing the problem to the numerical evaluation of eigenvalues and eigenfunctions of the transformed Hamiltonian.

\section{DISCUSSION}

The master equation (37) is mathematically exact and equivalent to the Liouville - von Neumann equation with the initial Hamiltonian (1)-(4). According to the master equation 
(37), the density matrix in the $Q$ subspace, $\rho^{(Q)}(t) \equiv \operatorname{Tr}_{(R)}\{\rho(t)\}$, does not experience dissipation. Indeed,

$$
\partial_{t} \rho^{(Q)}(t)=-i\left[H_{\Sigma}^{(Q)}, \rho^{(Q)}(t)\right]
$$

because the contributions due to $H_{S}^{(R)}$ and $\Re(t)$ are traced out (compare with Ref. [19]). The decoherence-free subspace [54] hence spans the entire $Q$ subspace. Physically, this is a striking manifestation of quantum interference. Analogous effects have recently been studied in $[55]$.

The presence of the decoherence-free $Q$ subspace cannot be ubiquitous in real aggregates. The key simplifying assumption is that all monomers are coupled to a single harmonic bath (the Hamiltonian (44)). More generally, we can assume that each monomer is coupled to its own bath,

$$
H_{S B}+H_{B}=\sum_{i=1}^{N_{B}} \sum_{k=1}^{N}\left(\frac{p_{i, k}^{2}}{2 m_{i}}+\frac{m_{i} \omega_{i}^{2}}{2}\left(x_{i, k}-\frac{c_{i} X_{k}}{m_{i} \omega_{i}^{2}}\right)^{2}\right) .
$$

The influence of these baths on the system dynamics is determined by the spectral density $g_{j k}(\omega)(1 \leq j, k \leq N$ enumerate different monomers). Apparently, the single bath (4) corresponds to $g_{j k}(\omega)=g(\omega)$ given by Eq. (5) and is thus equivalent to $N$ fully correlated baths. Uncorrelated baths yield $g_{j k}(\omega)=g(\omega) \delta_{j k}$. Partially correlated baths can also be defined by introducing the correlation parameter $0 \leq \gamma \leq 1$ as follows: $g_{j k}(\omega)=g(\omega)\left(\delta_{j k}+\gamma\left(1-\delta_{j k}\right)\right)$. The fully correlated and uncorrelated baths correspond to $\gamma=1$ and 0 , respectively. If $\gamma \neq 1$, no decoherence-free subspaces exist and the entire system relaxes to equilibrium. The above analysis suggests that the relaxation in the $R$ subspace has a characteristic rate $\nu \sim g(\omega)$, while the relaxation in the $Q$ subspace has a rate $\nu_{\gamma} \sim \nu(1-\gamma)$.

It is impossible to derive an exact master equation in the case of partially correlated baths. However, the master equation (37) may be augmented with phenomenological dissipative operators which describe relaxation in the $Q$ subspace. This can be done, for example, by switching to the eigenvalue representation in the $Q$ subspace and introducing the corresponding Redfield operator. Another option is to use the dissipation operator $-\nu_{\gamma}\left(1-\rho_{e q}^{(Q)} \operatorname{Tr}_{(Q)}\{\ldots\}\right)[56]$. Here $\rho_{e q}^{(Q)}$ is the equilibrium Boltzmann distribution corresponding to the Hamiltonian $H_{\Sigma}^{(Q)}$.

The problem of bath correlations can be analyzed from a microscopic perspective [57]. If the harmonic potentials in the Hamiltonians $H_{S B}$ and $H_{B}$ are considered as linearizations of anharmonic interaction potentials between the particles of the system and the bath, one 
arrives at the Hamiltonian (44), because the identical particles of the system should interact via the same (in our case, harmonic) potentials with the bath particles. The domain of validity of such a primitive linearization of the interaction potentials is, however, limited to short times. On the other hand, we can consider the harmonic bath and the bilinear system-bath coupling in the spirit of a normal mode analysis [58, 59]. Each particle of the system then experiences different local potentials from the bath particles, no matter whether the particles are identical or not. This is tantamount to introducing different (possibly correlated) local harmonic baths for each monomer, which ensure relaxation of all ( $Q$ and $R$ ) degrees of freedom. On physical grounds, one expects that intramolecular vibrational baths should be close to the fully correlated limit, while environmental vibrational baths should be less correlated.

Uncorrelated baths are the default choice in many simulations, although there exist strong experimental indications that this assumption is not universally applicable [32 34, 60, 61], and partially correlated baths are more appropriate for describing coherent energy and exciton transport $[62-76]$. The present analysis suggests that relaxation of molecular aggregates in the $R$ and $Q$ subspaces is governed by two different rates $\nu$ and $\nu_{\gamma}$. For fully correlated baths, $\nu_{\gamma}=0$. One hence expects that $\nu_{\gamma}<\nu$ for partially correlated baths. The slow relaxation in the $Q$ subspace manifests itself in slow decays of the populations and coherences of the aggregate density matrix. This may be one of the reasons of the existence of long-lived coherent optical responses in dissipative excitonic systems [32 41]].

\section{ACKNOWLEDGMENTS}

This work has been supported by the Deutsche Forschungsgemeinschaft (DFG) through a research grant and the DFG-Cluster of Excellence "Munich-Centre for Advanced Photonics" (www.munich-photonics.de). We wish to thank Sergy Grebenshchikov, Leah Z. Sharp, and Michael Thoss for helpful discussions.

[1] U. Weiss, Quantum Dissipative Systems, 2nd ed. (World Scientific, Singapore, 1999).

[2] H. Spohn, Rev. Mod. Phys. 52, 569 (1980). 
[3] A. J. Leggett, S. Chakravarty, A. T. Dorsey, Matthew P. A. Fisher, Anupam Garg, and W. Zwerger, Rev. Mod. Phys. 59, 1 (1987).

[4] T. Renger, V. May, O. Kühn, Phys. Rep. 343 (2001) 137.

[5] Y. Yan and R. Xu, Annu. Rev. Phys. Schem. 56, 187 (2005).

[6] A. Rivas, A. D. K. Plato, S. F. Huelga, and M. B. Plenio, New J. Phys. 12, 113032 (2010).

[7] W. M. Zhang, T. Meier, V. Chernyak, and S. Mukamel, J. Chem. Phys. 108, 7763 (1998).

[8] A. G. Redfield, Adv. Magn. Reson. 1, 1 (1965).

[9] J. Ankerhold, P. Pechukas, and H. Grabert, Phys. Rev. Lett. 87, 086802 (2001).

[10] W. T. Coffey, Yu. P. Kalmykov, S. V. Titov, and B. P. Mulligan, Phys. Chem. Chem. Phys. 9, 3361 (2007).

[11] Y. Tanimura, J. Phys. Soc. Jpn. 75, 082001 (2006).

[12] R. Doll, D. Zueco, M. Wubs, S. Kohler, and P. Hänggi, Chem. Phys. 347, 243 (2008).

[13] F. Haake and R. Reibold, Phys. Rev. A 32, 2462 (1985).

[14] B. L. Hu, J. P. Paz, and Y. Zhang, Phys. Rev. D 45, 2843 (1992).

[15] J. J. Halliwell and T. Yu, Phys. Rev. D 53, 2012 (1996).

[16] R. Karrlein and H. Grabert, Phys. Rev. E 55, 153 (1997).

[17] J.-H. An and W.-M. Zhang, Phys. Rev. A 76, 042127 (2007).

[18] C. H. Chou, T. Yu, and B. L. Hu, Phys. Rev. E 77, 011112 (2008).

[19] C. H. Chou, B. L. Hu, and T. Yu, Physica A 387, 432 (2008).

[20] C. W. Gardiner, Optics Communications 243, 57 (2004).

[21] T. Prosen and B. Zunkovič, New J. Phys. 025016 (2010).

[22] M. F. Gelin and D. S. Kosov, Phys. Rev. E 80, 022101 (2009).

[23] A. G. Dijkstra and Y. Tanimura, New J. Phys. 12, 055005 (2010).

[24] T. Dittrich, P. Hänggi, G.-L. Ingold, B. Kramer, G. Schön, and W. Zwerger, Quantum transport and dissipation. (Wiley-VCH, 1998).

[25] G. D. Mahan, Many Particle Physics. 3rd ed. (Springer, 2000).

[26] M. Richter, K. J. Ahn, A. Knorr, A. Schliwa, D. Bimberg, M. E. A. Madjet, and T. Renger, phys. stat. sol. (b) 243, 2302 (2006).

[27] D. Abramavicius, B. Palmieri, D. V.Voronine, F. Sanda, and S. Mukamel. Chem. Rev. 109, $2350(2009)$.

[28] R. J. Baxter, Exactly Solved Models in Statistical Mechanics (Academic Press Inc, London, 
1992).

[29] J. Roden, W. T. Strunz, and A. Eisfeld, J. Chem. Phys. 134, 034902 (2011).

[30] M. Galperin, M. A. Ratner, A. Nitzan, J. Phys.: Condens. Matter 19, 103201 (2007).

[31] R. Härtle and M. Thoss, Phys. Rev. B 83, 115414 (2011).

[32] H. Lee, Y.-C. Cheng, and G. R. Fleming, Science 316, 1462 (2007).

[33] J. M. Womick, S. A. Miller, and A. M. Moran, J. Phys. Chem. B 113, 6630 (2009).

[34] E. Collini and G. D. Scholes, Science 323, 369 (2009).

[35] T. Bittner, K.-D. Irrgang, G. Renger, and M. R. Wasielewski, J. Phys. Chem. 98, 11821 (1994).

[36] I. Yamazaki, N. Aratari, S. Akimoto, T. Yamazaki, and A. Osuka, J. Am. Chem. Soc. 125, 7192 (2003).

[37] S. Savikhin, D. R. Buck, and W. S. Struve, Chem. Phys. 223, 303 (1997).

[38] G. S. Engel, T. R. Calhoun, E. L. Read, T.-K. Ahn, T. Mančal, Y.-C. Cheng, R. E. Blankenship, and G. R. Fleming, Nature 446, 782 (2007).

[39] F. Milota, J. Sperling, A. Nemeth, and H. F. Kauffmann, Chem. Phys. 357, 45 (2009).

[40] G. Panitchayangkoona, D. Hayesa, K. A. Fransteda, J. R. Carama, E. Harela, J. Wenb, R. E. Blankenshipb, and G. S. Engel, Proc. Natl. Acad. Sci. USA 107, 12766 (2010).

[41] E. Collini, C. Y. Wong, K. E. Wilk, P. M. G. Curmi, P. Brumer, and G. D. Scholes, Nature $463,644(2010)$.

[42] D. Egorova, M. F. Gelin, M. Thoss, H. Wang, and W. Domcke, J. Chem. Phys. 129, 214303 (2008).

[43] M. F. Gelin, D. Egorova, and W. Domcke, J. Chem. Phys. 131, 124505 (2009).

[44] A. Garg, J. N. Onuchic and V. Ambegaokar, J. Chem. Phys. 83, 4491 (1985).

[45] V. Chernyak and S. Mukamel, J. Chem. Phys. 105, 4565 (1996).

[46] K. H. Hughes, C. D. Christ, and I. Burghardt, J. Chem. Phys. 131, 024109 (2009).

[47] K. H. Hughes, C. D. Christ, and I. Burghardt, J. Chem. Phys. 131, 124108 (2009).

[48] A. Nemeth, F. Milota, T. Mančal, T. Pullerits, J. Sperling, J. Hauer, H. F. Kauffmann, and N. Christensson, J. Chem. Phys. 133, 094505 (2010).

[49] R. Zwanzig, J. Chem. Phys. 60, 2717 (1974).

[50] M. F. Gelin, D. Egorova and W. Domcke, J. Phys. Chem. B 115, 5648 (2011).

[51] J. Seibt and V. Engel, Chem. Phys. 347, 120 (2008).

[52] M. Wagner, J. Phys. A.: Math. Gen. 17, 2319 (1984). 
[53] H. Eiermann and M. Wagner, J. Chem. Phys. 105, 6713 (1996).

[54] D. A. Lidar, I. L. Chuang, and K. B. Whaley, Phys. Rev. Lett. 81, 2594 (1998).

[55] F. Caruso, A. W. Chin, A. Datta, S. F. Huegla, and M. B. Plenio, J. Chem. Phys. 131, 105106 (2009).

[56] M. F. Gelin, D. Egorova and W. Domcke, J. Chem. Phys. 118, 5287 (2003).

[57] M. F. Gelin and M. Thoss, Phys. Rev. E 79, 051121 (2009).

[58] G. Goodyear and R. M. Stratt, J. Chem. Phys. 105, 10050 (1996).

[59] G. Goodyear and R. M. Stratt, J. Chem. Phys. 107, 3098 (1997).

[60] N. Demirdöven, M. Khalil, A. Tokmakoff, Phys. Rev. Lett. 89, 237401 (2002).

[61] P. G. Wolynes, Proc. Natl. Acad. Sci. USA 106, 17247 (2009).

[62] T. Renger and R. A. Marcus, J. Chem. Phys. 116, 9997 (2002).

[63] J. Adolphs and T. Renger, Biophys. J. 91, 2778 (2006).

[64] M. Cho, H. M. Vaswani, T. Brixner, J. Stenger, and G. R. Fleming, J. Phys. Chem. B 109, $10542(2005)$.

[65] P. Nalbach, J. Eckel, and M. Thorwart, New. J. Phys. 12, 065043 (2010).

[66] A. Ishizaki and G. R. Fleming, New J. Phys. 12, 055004 (2010).

[67] F. Caruso, A. W. Chin, A. Datta, S. F. Huegla, and M. B. Plenio, Phys. Rev. A 81, 062346 (2010).

[68] C. Olbrich, J. Strümpfer, K. Schulten, and U. Kleinekathöfer, J. Phys. Chem. B 115, 758 (2011).

[69] J. Strümpfer and K. Schulten, J. Chem. Phys. 134, 095102 (2011).

[70] R. Venkatramani and S. Mukamel, J. Chem. Phys. 117, 11089 (2002).

[71] D. Abramavicius and S. Mukamel, J. Chem. Phys. 133, 064510 (2010).

[72] D. Abramavicius and S. Mukamel, J. Chem. Phys. 134, 174504 (2011).

[73] A. Ishizaki and Y. Tanimura, J. Phys. Chem. A 111, 9269 (2007).

[74] A. Perdomo, L. Vogt, A. Najmaie, and A. Aspuru-Guzik, Appl. Phys. Lett. 96, 093114 (2010).

[75] F. Fassioli, A. Nazir, and A. Olaya-Castro, J. Phys. Chem. Lett. 1, 2139 (2010).

[76] M. Sarovar, Y.-C. Cheng, K. B. Whaley, Phys. Rev. E 83, 011906 (2011). 